J. Clin. Chem. Clin. Biochem.

Vol. 15, 1977, pp. 101-108

\title{
Ein neues Verfahren zur Herstellung linearer Polyacrylamidgradienten
}

\author{
Von $K$. Lorentz und Barbara Flatter
}

\author{
Aus der I. Medizinischen Klinik der Medizinischen Hochschule Lübeck
}

(Eingegangen am 10. Juni/8. November 1976)

Zusammenfassung: Lineare Konzentrationsgradienten verbessern die elektrophoretische Trennung in Polyacrylamidgelen. Sie entstehen durch Überlagerung gleicher Teile konzentrierter und verdünnter Monomerlösung (180-260 bzw. 23-50 g/l Acrylamid) in Gelröhrchen, die um 3-10 ${ }^{\circ}$ gegenüber der Waagerechten geneigt sind, und so anschließend, bis zur homogenen Mischung der berührenden Teile beider Ansätze, um ihre Längsachsen rotiert werden. Darauf polymerisiert man in senkrechter Stellung. Der dichtere Anteil en thält 4-Nitrophenol, so daß die Polymerkonzentration pro Gelsegment densitometrisch zu bestimmen ist.

Aufbau und Gebrauch der Drehvorrichtung sind einfach und liefern gut reproduzierbare Ergebnisse. Das vielseitige Verfahren eignet sich auch für die Trennung von Lipoproteinen und Enzymen und liefert bei gleichzeitiger Anwendung eines pH-Gradienten 27-30 Proteinfraktionen im Serum.

\section{A new method for the preparation of linear polyacrylamide gradients}

Summary: Continuous linear gradients improve electrophoretic separations in polyacrylamide. They are obtained by overlayering equal volumes of two monomer solutions with different acrylamide concentrations, namely $180-260$ and $23-50 \mathrm{~g} / \mathrm{l}$, in the usual gel tubes. The tubes are tilted $3-10^{\circ}$ to the horizontal, and rotated around their longitudinal axis until the parts of both solutions in contact are mixed homogeneously. Subsequent polymerization takes place in the vertical position. The more concentrated lower solution contains 4-nitrophenol, so that the final polymer concentration at any point of the gel can be measured densitometrically.

The construction and handling of the rotation device are simple and yield highly reproducible results. This versatile method was evaluated for the separation of proteins, lipoproteins, and enzymes in human serum and yields 27-30 protein fractions by additional use of a pH-gradient.

\section{Einfiibrung}

Wegen ihres höheren Molekülsiebeffektes trennen kontinuierliche Gradienten aus Polyàcrylamid bei der Elektrophorese Proteingemische prinzipiell besser als Gele einheitlicher Porengröße $(1,2)$. Ihre Herstellung erfordert jedoch eine Mischapparatur $(2,3)$ mit entsprechendem Mehrbedarf an Reagenzien, oder sie beschränkt sich auf Mikromethoden (4). Anderenfalls müssen Fertiggele invariabler Zusammensetzung $(5,6)$ benutzt werden. Daher enthält dieșe Mitteilung ein einfaches Verfahren zum Ansatz linearer Gelgradienten beliebiger Konzentration und Steilheit.

\section{Material und Methoden}

\section{Geräte}

Wir polymerisieren die Gele in $85 \mathrm{~mm}$ langen Röhrchèn àus Duranglas mit einem Innendurchmesser von $4,8 \mathrm{~mm}$ und führen alle Trennungen mit dẹr Apparatự „StandardsPA “
(Biomol, Ilvesheim) durch. Fabrikate mit zylindrischen Einsätzen für Gelröhrchen (z. B. Boskamp, Pleuger, WTW) sind möglicherweise besser geeignet, da sie durch Einsetzen einer zentralen Achse die Verwendung der nachstehend beschriebenen Vorrichtung erübrigen. Sie besteht aus zwei, etwa $2 \mathrm{~mm}$ dicken Scheiben Polycarbonat (Makrolon) oder Polymethacrylat (Plexiglas), die man übereinanderlegt, zentral durchbohrt und kreisförmig mit 6-12 Löchern, deren Größe dem äußeren Durchmesser der Röhrchen entspricht, versieht. Ihr Abstand voneinander und vom Zentrum hat keinen Einfluß auf die Ausbildung des Gelgradienten. Man stanzt sie am besten mit einem heißen Korkbohrer als circulär angeordnete Reihe einander berührender Halbkreise. Dann schiebt man die beiden (z. B. sternförmig gezackten) Scheiben, $40-60 \mathrm{~mm}$ voneinander entfernt, direkt auf einen Glas- oder Metallstab, der als Achse eines Rührmotors (z. B. Heidolph) dient, oder man klebt sie zuvor auf die Enden eines abgeschnittenen Polycarbonat- oder Polyethylenröhrchens (Abb. 1), die dazu mit Tetrahydrofuran benetzt wurden. Dabei ist die absolut deckungsgleiche Anordnung der Löcher entscheidend, um eine exakt achsenparallele Ausrichtung der Röhrchen żu sichern (Abb. 2).

Die Form des Gradienten läßt sich bei $405 \mathrm{~nm}$ densitometrisch mittels Pherogrammauswerter 2602, Photometer Eppendorf $1101 \mathrm{M}$ und Kompensationsschreiber 4412 (sämtlich von Netheler \& Hinz, Hamburg) aufzeichnen. Die Zymogramme 


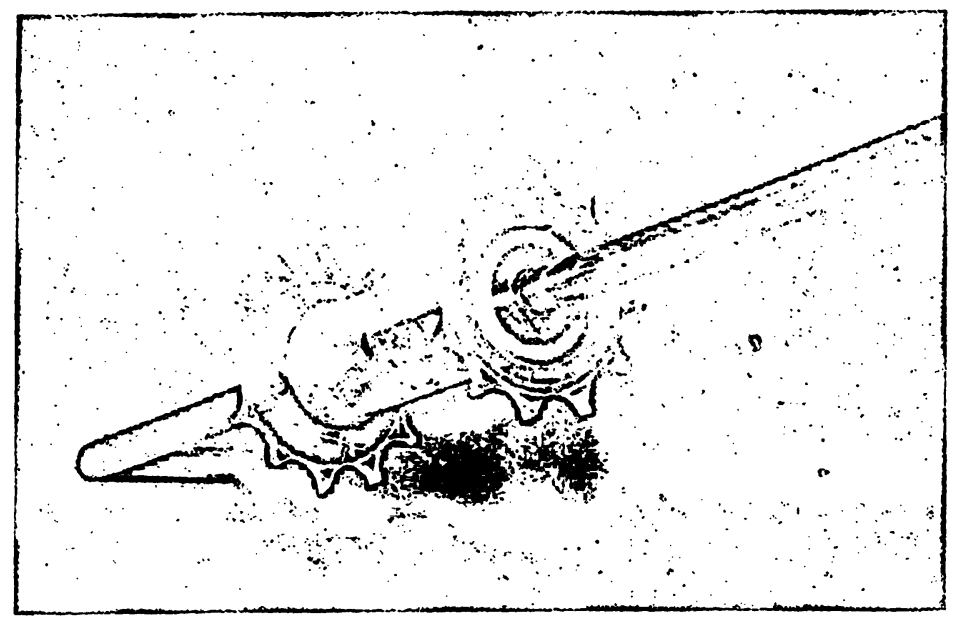

Abb. 1. Träger zur Herstellung zylindrischer Gradientengele in Rotationsstellung.

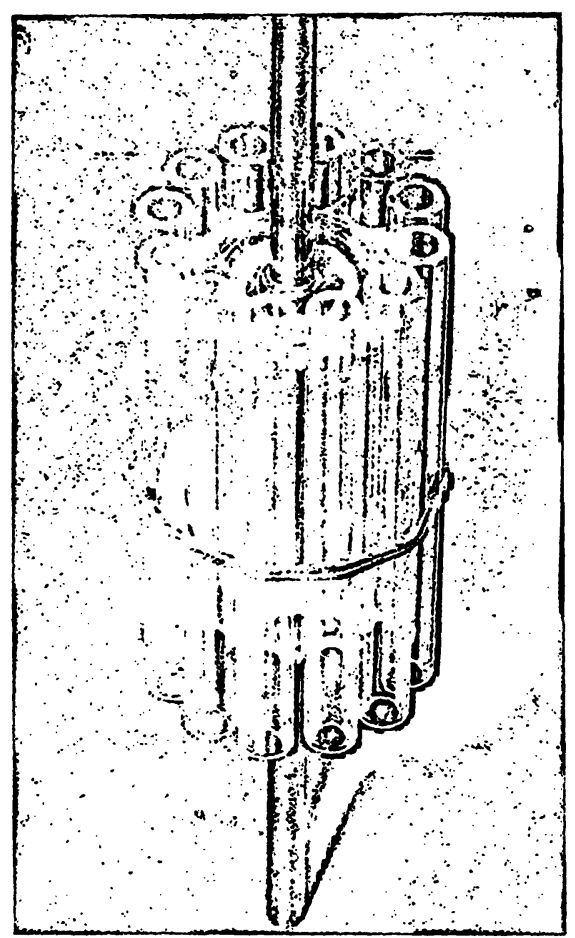

Abb. 2. Vorrichtung mit Röhrchen in Polymerisationsposition.

und Proteinpherogramme kann man mit der Kombination Spektralphotometer PMQ 3, Disc-Ansatz ZK 4 (beide von Zeiß, Oberkochen) und Schreiber Servogor S (Metrawatt, Nürnberg) auswerten.

\section{Reagenzien}

Zur Identifizierung der Serumproteine dienten M- und LCPartigen Immunodiffusionsplatten der Behringwerke AG (Frankfurt/M.). Farbstoffe bezogen wir von Serva (Heidelberg) und Chroma (Stuttgart). Alle anderen Reagenzien lieferte E. Merck (Darmstadt) in bestmöglicher Qualität. Acrylamid wurde in Chloroform, Methylenbisacrylamid in Aceton umkristallisiert. Wir setzten alle Lösungen, wenn nicht anders vermerkt, mit demineralisiertem Wasser an.

Die in der Folge beschriebenen Ansätze erwiesen sich nach Vorversuchen als bestgeeignet für die Fraktionierung von Serum- proteinen. Sie können zur Trennung anderer Proteingemische verändert werden, doch sollte das molare Verhältnis von Acrylamid $z$ u BIS ${ }^{1}$ ) immer 75:1 betragen, und die Totalkonzentration beider darf $32 \mathrm{~g} / 1$ nicht unterschreiten, damit klare elastische Gele von ausreichender Festigkeit entstehen. Auch das äquimolare Verhältnis der Katalysatoren und deren absolute Konzentration sind als optimal anzusehen.

Elektrodenpuffer (Tris 45,5 mmol/1, Glycin $192 \mathrm{mmol} / \mathrm{l}$, pH 8,6, lonenstärke 0,118): 5,5 g Tris und 14,4 g Glycin auf $1000 \mathrm{ml}$ lösen.

Gelpuffer (Tris 1,5 mol/l, Schwefelsäure $100 \mathrm{mmol} / \mathrm{l}, \mathrm{pH} \mathrm{8,6):}$ $182 \mathrm{~g}$ Tris in etwa $800 \mathrm{ml}$ Wasser mit $100 \mathrm{ml}$ Schwefelsäure $1 \mathrm{~mol} / \mathrm{l}$ auf pH 8,6 einstellen und ad $1000 \mathrm{ml}$ auffüllen.

Katalysator 1 (Temed $30 \mathrm{mmol} / \mathrm{l}$, Tris $1,5 \mathrm{~mol} / \mathrm{l}$, Schwefelsäure $100 \mathrm{mmol} / \mathrm{l}, \mathrm{pH} 8,6): 4,5 \mathrm{ml}$ Temed mit Gelpuffer auf $1000 \mathrm{ml}$ verdünnen.

Katalysator 2 (Ammoniumperoxodisulfat $30 \mathrm{mmól} / \mathrm{l}$ ): $680 \mathrm{mg}$ $\left(\mathrm{NH}_{4}\right)_{2} \mathrm{~S}_{2} \mathrm{O}_{8}$ auf $100 \mathrm{ml}$ lösen.

Monomer $A$ (Acrylamid $5,49 \mathrm{~mol} / \mathrm{l}$, BIS $73 \mathrm{mmol} / \mathrm{l}$, Kaliumhexacyanoferrat (III) $300 \mu \mathrm{mol} / \mathrm{l}$ ): $390 \mathrm{~g}$ Acrylamid, $11,2 \mathrm{~g}$ BIS und $100 \mathrm{mg} \mathrm{K} 3\left[\mathrm{Fe}(\mathrm{CN})_{6}\right]$ ad $1000 \mathrm{ml}$ lösen.

Monomer B (Acrylamid $845 \mathrm{mmol} / \mathrm{l}$, BIS $11 \mathrm{mmol} / \mathrm{l}$, Kaliumhexacyanoferrat (III) $334 \mu \mathrm{mol} / \mathrm{l}$ ): $60 \mathrm{~g}$ Acrylamid, $1,72 \mathrm{~g}$ BIS und $110 \mathrm{mg} \mathrm{K} 3\left[\mathrm{Fe}(\mathrm{CN})_{6}\right]$ auf $1000 \mathrm{ml}$ lösen.

Katalysator 2 nur $8 \mathrm{~h}$, übrige Lösungen $5 \mathrm{~d}$ bei $4{ }^{\circ} \mathrm{C}$ haltbar. 4-Nitrophenol (7,19 mmol/l in Methanol 1,72 mol/l): $100 \mathrm{mg}$ 4-Nitrophenol in $10 \mathrm{ml}$ Methanol lösen und mit demineralisiertem Wasser auf $100 \mathrm{ml}$ verdünnen.

Zur Gelbereitung jeweils $1 \mathrm{Tl}$. Katalysator 1 und $2 \mathrm{mit} 3 \mathrm{Tln}$. Monomer A oder B mischen. Dem Ansatz A (234 g Acrylamid/l) werden 100-120 $\mu$ l Nitrophenollösung auf $10 \mathrm{ml}$ zur Markierung zugesetzt.

Bromphenolblau $(14,9 \mathrm{mmol} / \mathrm{l}=10 \mathrm{~g} / \mathrm{l}$ Ethanol).

Färbelösung (Amidoschwarz 10 B 8,1 mmol/1, 5-Sulfosalicylsäure $590 \mathrm{mmol} / \mathrm{l}$, Methanol 4,93 mol/l): $5 \mathrm{~g} \mathrm{Amidoschwarz,}$ $150 \mathrm{~g}$ Sulfosalicylsäure (Dịydrat) und $200 \mathrm{ml}$ Methanol ad $1000 \mathrm{ml}$ auffüllen und filtrieren.

Essigsäure (1,75 mol$/ 1)$ - Methanol (6,2 mol/1): $100 \mathrm{ml}$ Essigsäure und $250 \mathrm{ml}$ Methanol ad $1000 \mathrm{ml}$ verdünnen.

Dichlordimethylsilan (415 mmol/1 = $50 \mathrm{ml} / 1$ Benzol).

Tritonlösung (Triton X-100 $5 \mathrm{~g} / \mathrm{l}$ ).

1) Benutzte Abkürzungen:

BIS $\quad \mathrm{N}, \mathrm{N}^{\prime}$-Methylenbisacrylamid

Temed $\mathbf{N}, \mathbf{N}, \mathbf{N}^{\prime}, \mathrm{N}^{\prime}$-Tetramethylethylendiamin

TI(e) Volumenteil(e) 


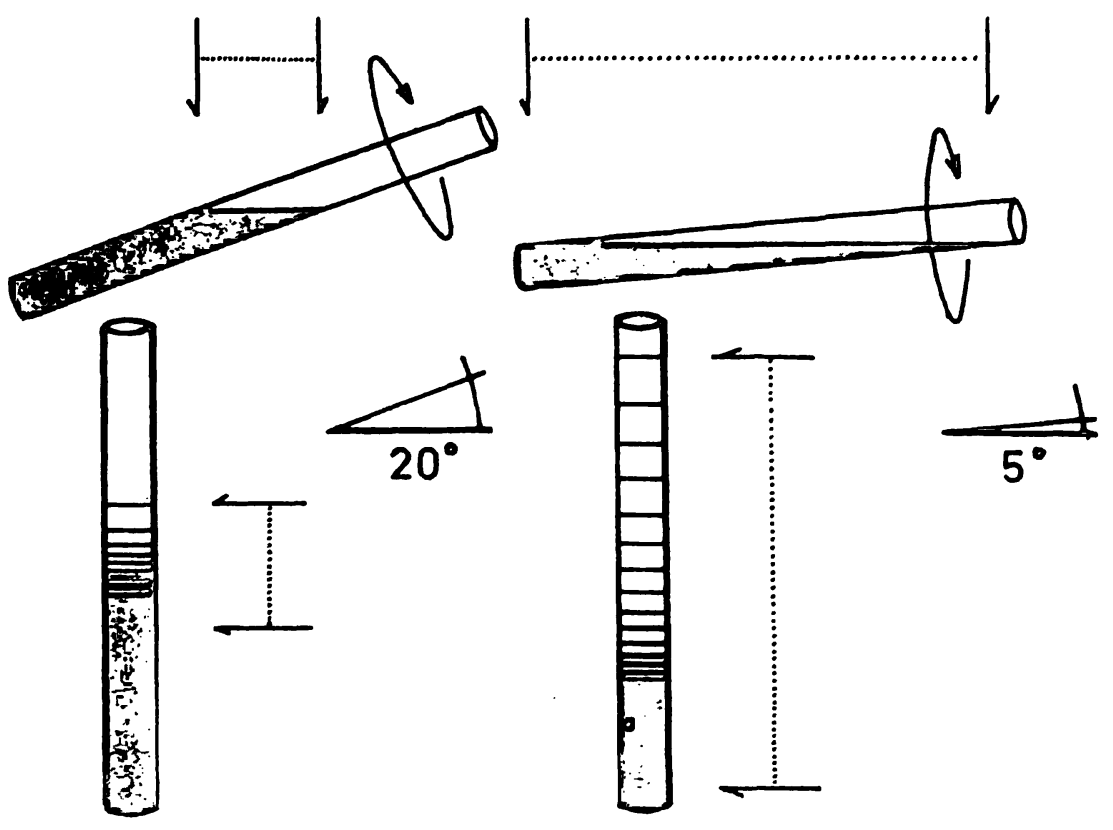

Abb. 3. Entstehung linearer Gelgradienten durch Rotation unterschiedlich dichter Ansätze bei $5^{\circ}$ bzw. $20^{\circ}$ Neigung mit Polymerisation in der Senkrechten. Der Abstand zwischen den Pfeilen bezeichnet die Länge des Gradienten.

\section{Methoden}

Die mit Chromschwefelsäure und Aceton gereinigten Röhrchen werden in Dichlordimethylsilan getaucht, bei $60^{\circ} \mathrm{C}$ getrocknet (7) und mit Gummiringen senkrecht in die Drehvorrichtung gespannt (Abb. 2). Man füllt sie wegen der minimalen Wandhaftung aus einer Spritze mittels Kanüle oder Polyethylenschlauch jeweils aufsteigend $38 \mathrm{~mm}$ hoch nacheinander mit den Ansätzen $A\left(d_{4}^{20} 1,015\right)$ und $B\left(d_{4}^{20} 1,008\right)$. Dann neigt man die Achse auf $5^{\circ}$ oder $10^{\circ}$ gegenüber der Horizontalen und wartet $1 \mathrm{~min}$. Die Silanisierung erlaubt auch bei dieser Neigung eine einwandfreie Überlagerung beider Gelmischungen (Abb. 3). Anschließend wird $10 \mathrm{~min}$ mit $54 \mathrm{U} / \mathrm{min}$ gedreht, wodurch eine homogene Mischung beider Ansätze im Verhältnis ihrer in der Querschnittsebene des Röhrchens bewegten Volumina eintritt. Dadurch bildet sich ein kontinuierlicher, nach Neigungswinkel unterschiedlich langer, Gradient aus. Infolge der nach abwärts zunehmenden Dichte bleibt er in senkrechter Stellung, in der nicht rotiert wird, stabil (Abb. 3). Die Röhrchen werden dann, zur Ableitung der Polymerisationswärme aus dem dichten Gel, etwa $70 \mathrm{~mm}$ tief in Wasser von Raumtemperatur eingetaucht (Becherglas mit Rührmagnet). Wegen der geringen Monomerkonzentration im oberen Gel ist eine Überschichtung mit Wasser überflüssig, doch sollten die Gele wie bei Mikroverfahren (4) noch 4 bis $24 \mathrm{~h}$ verschlossen bei Raumtemperatur lagern.

Um die Totalkonzentration $(\mathrm{g} / \mathrm{l})$ an Polyacrylamid für jeden Abschnitt des Gradienten zu berechnen, legt man mit den verwendeten Monomeren einmalig eine Standardgerade an. Dazu mischt man wechselnde, aber komplementäre Volumina der Ansätze A und B und polymerisiert sie in Röhrchen, die gegen ein Röhrchen mit Gel B (hier Polyacrylamid-Gesamtkonzen: tration $37 \mathrm{~g} / \mathrm{l}=\mathbf{A}_{405} \mathrm{~nm}$ 0,000) oder gegen Luft photometriert werden. Die der Absorption von 4-Nitrophenol entsprechende Polymerkonzentration berechnet man aus den Anteilen von $\mathbf{A}$ und B im Gemisch (Tab. 1). Diese Werte lassen sich in jede Densitometriekurve übertragen. Hierzu wird das weitporige Ende des Gels mit dem Photogrammauswerter (Blende $3 \times 0,5 \mathrm{~mm}$ ) wie oben auf $A=0,000$ eingestellt. Am Kompensationsschreiber wählt man zum Vorschub des Auswerters von $25 \mathrm{~mm} / \mathrm{min}$ die Papiergeschwindigkeit $v=50 \mathrm{~mm} / \mathrm{min}$ und den Bereich $A_{0-1,0}=200$ $\mathrm{mm}$, so daß sich bei Zunahme der Polyacrylamid-Gesamtkonzentration um $20 \mathrm{~g} / 1$ (etwa $20 \mathrm{~mm}$ ) pro $10 \mathrm{~mm}$ Gel eine Steigung der Absorptionsgeraden um $45^{\circ}$ ergibt (Abb. 4).

Zur Proteinfraktionierung werden $3 \mu \mathrm{l}$, zur Darstellung von Lipiden und Enzymen 5-20 $\mu$ l Serum aufgesetzt. Bei Magensaft
Tab. 1. Ansatz, Totalkonzentration und Absorption verschiedener Gele bei Lagerung nach Abschluß der Polymerisation. Messungen gegen Luft, $n=11$ Werte pro Röhrchen.

\begin{tabular}{|c|c|c|c|c|c|c|}
\hline $\begin{array}{l}\text { Tle. } \\
\text { A B }\end{array}$ & $\begin{array}{l}\text { Polyacrylamid- } \\
\text { Gesamtkonzen- } \\
\text { tration }[\mathrm{g} / \mathrm{l}]\end{array}$ & $\begin{array}{l}\text { A } 405 \mathrm{~nm} \\
\frac{1}{\mathrm{x}} \mathrm{h}\end{array}$ & $s$ & $\frac{2}{x} h$ & $\frac{3}{x} h$ & $\frac{12}{x} h$ \\
\hline $\begin{array}{ll}- & 4 \\
1 & 3 \\
2 & 2 \\
3 & 1 \\
4 & -\end{array}$ & $\begin{array}{r}37,0 \\
87,9 \\
138,9 \\
189,8 \\
240,7\end{array}$ & $\begin{array}{l}0,058 \\
0,290 \\
0,512 \\
0,756 \\
0,997\end{array}$ & $\begin{array}{l}0,002 \\
0,006 \\
0,010 \\
0,020 \\
0,018\end{array}$ & $\begin{array}{l}0,058 \\
0,288 \\
0,509 \\
0,750 \\
0,985\end{array}$ & $\begin{array}{l}0,058 \\
0,286 \\
0,504 \\
0,743 \\
0,978\end{array}$ & $\begin{array}{l}0,057 \\
0,281 \\
0,496 \\
0,734 \\
0,965\end{array}$ \\
\hline
\end{tabular}

gibt man $50 \mu$ unter Zusatz von etwas Saccharose in Substanz oder besser $5 \mu$ l einer auf $0,1 \mathrm{Tl}$., bei Liquor $5 \mu \mathrm{l}$ einer auf 0,02 Tle. des Ausgangsvolumens eingeengten Probe auf. Nach Markieren des Kathodenpuffers mit 20-50 $\mu$ Bromphenolblaulösung wird $2 \mathrm{~h}$ mit $30 \mathrm{~V}$, dann etwa $18 \mathrm{~h}$ mit $50 \mathrm{~V}$ getrennt. $\mathrm{Da}$ nach dieser Zeit die Fraktionen praktisch nicht mehr wandern, entfernt man die Gele anschließend durch Anspülen mit der Tritonlösung mittels Spritze und dünner Kanüle vom kathodischen Ende bis zur markierten Albuminbande. Die gelockerten Gele lassen sich darauf leicht mit einer Pipettierhilfe (Brand, Wertheim) oder einem Peleusball auspressen. Sie werden durch die Farblösung in $1 \mathrm{~h}$ gleichzeitig fixiert und gefärbt. Um eine Volumenänderung zu verhindern, entfärbt man unter wiederholtem Wechsel der Lösung in Essigsäure-Methanol, sonst in Essigsäure $70 \mathrm{~g} / \mathrm{l}$.

Die Lipoproteine des Serums werden nach Allen (8) mit Sudanschwarz vorgefärbt, in einem Gradientengel $23,4-234 \mathrm{~g} / \mathrm{l}$ getrennt, und zur Auswertung im Röhrchen belassen. Für Magensaft- und Liquorproben verwendet man, bei Konstanz der übrigen Parameter, besser einen flachen Gradienten von 50-216 g/l. Lactatdehydrogenase wird nach Dietz \& Lubrano (9), Esterase durch $30 \mathrm{~min}$ Inkubation bei $25^{\circ} \mathrm{C}$ in $100 \mathrm{mmol} / \mathrm{l}$ Phosphat pH 7,0 mit Essigsäure-3-indolylester $100 \mu \mathrm{mol} / 1$ oder Naphthyl-2-acetat $4,3 \mathrm{mmol} / 1$ und Echtblausalz $10 \mathrm{~B} 1 \mathrm{~g} / \mathrm{l}$ nachgewiesen. Alkalische Phosphatase und Arylamidase werden nach Fraktionierung in Tris (165 mmol/l) - Borat (11,5 mmol/l) - Elektrodenpuffer ( $\mathrm{pH} \mathrm{8,6)}$ in üblicher Weise dargestellt $(10$, 11). - Außerdem verglichen wir die beschriebene Anfärbung 


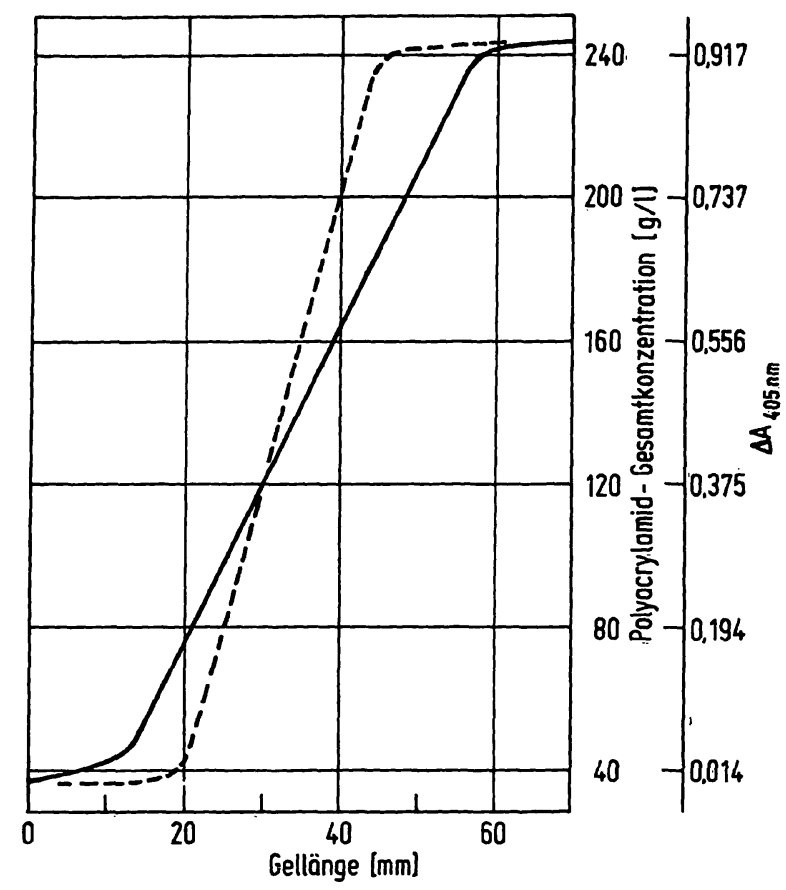

Abb. 4. Densitogramme von Gradientengelen in Duranglasröhrchen $85 \times 4,8 \mathrm{~mm}$ bei $5^{\circ}(-)$ und $10^{\circ}(--)$ Rotationswinkel $3 \mathrm{~h}$ nach abgeschlossener Polymerisation. Abszisse: Gellänge $(10 \mathrm{~mm}=20 \mathrm{~mm}$ Papiervorschub), Ordinate: $A_{0-1,0}=200 \mathrm{~mm}(20 \mathrm{~g} / \mathrm{l}$ Polyacrylamid-Gesamtkonzentration $20 \mathrm{~mm}$ ), s. Methoden.

der Proteine mit anderen Verfahren wie Uniblue nach Datyner \& Finnimore (12), Coomassie Brillantblau R 250 (13), Säureviolett $4 \mathrm{BL}$ bzw. 6B (14) und Ponceau S (beide in MethanolSulfosalicylsäure) und identifizierten die Fraktionen durch Segmentieren ungefärbter Gele. Die $1 \mathrm{~mm}$ dicken Scheibchen wurden mit $155 \mathrm{mmol} / \mathrm{l}$ Natriumchlorid zerrieben und auf Partigenplatten gebracht.

\section{Ergebnisse}

Reproduzierbarkeit und Form der Gelgradienten Die Prüfung des Verfahrens und seiner Bedingungen setzte einen Indikator für die Geldichte mit folgenden Eigenschaften voraus:

1. hohe anodische Beweglichkeit (negative Ladung), um das Gel vor den Proteinen und Bromphenolblau zu passieren,

2. hoher molarer Absorptionskoeffizient in einem Bereich des Spektrums, der nicht durch die Lichtabsorption der Gelmatrix beeinflußt wird,

3. fehlende Interferenz mit den Reaktanten und maximale Resistenz gegen Oxidation und Wärme bei der Polymerisation und

4. ausreichende Stabilität bei der Lagerung der Gele vor dem Trennvorgang.

4Nitrophenol erfüllte diese Bedingungen weitaus besser als eine Reihe von Azofarbstoffen (Amidoschwarz 10B,
Neucoccin, Chromotrop 2R). Die drei zuerst genannten Eigenschaften wurden bei der Elektrophorese und durch Photometrie bzw. Messung der Polymerisationszeit beurteilt, die letzte durch Densitometrie. Wir registrierten die Absorption der zum Anlegen der Standardkurve hergestellten Gelröhrchen zu verschiedenen Zeitpunkten nach der Polymerisation (Tab. 1). Bei linearer Abhängigkeit von $\mathrm{A}_{405}$ gegenüber der Polyacrylamid-Gesamtkonzentration ergab sich eine Abnahme der Absorption von maximal $3 \%$ nach $3 \mathrm{~h}$ bzw. $4,5 \%$, wenn man das $\Delta A$ gegenüber Gel B (A minus 0,058) zugrunde legte. Aufgrund dieser Daten wurde regelmäßig $3 \mathrm{~h}$ nach $\mathrm{Abschluß}$ der Polymerisation densitometriert, da bei weiterem Zuwarten durch Diffusion im feuchten Gel eine $\mathrm{Ab}$ flachung des unteren und oberen Wendepunktes (Abb. 4) und damit eine optische Verkürzung des linearen $\mathrm{Ab}$ schnitts eintrat. Unter den genannten Bedingüngen variierte die Steigung dieses Teils bei 12 Röhrchen derselben Präparation nur minimal und erreichte selbst bei 10 verschiedenen Einstellungen von $5^{\circ}$ Neigung der Rotationsachse nur 5\% Abweichung (Tab. 2). Dieser Winkel lieferte die besten Resultate. Länge, Anstieg, Form und Reproduzierbarkeit der Absorptionsgeraden hingen wesentlich von der lichten Weite und deren Toleranz bei verschiedenen Röhrchen ab. Bei Maximaldifferenzen zwischen 4,75 und $4,80 \mathrm{~mm}$, die einer Streuung von etwas über $1 \%$ entspricht, überschritt der aus den Standardabweichungen von Tảbelle 1 errechnete VK der Absorptionen nicht 2,7\%.

Wurde mit $54 \mathrm{U} / \mathrm{min}$ gearbeitet, so veränderten sich Länge und Anstieg des Gradienten nicht zwischen 5 und 15 min Rotationsdauer, doch wurde erst nach 7-8 min eine ruhige Linie des Schreibers als Anzeichen homogener Mischung erreicht. Bei Vorgabe einer konstanten Rotationsperiode von 10 min erhielten wir zwischen 45 und $65 \mathrm{U} / \mathrm{min}$ identische Ergebnisse. Entscheidend war hierfür die $1 \mathrm{~min}$ lange Ruhestellung des geneigten Röhrchens vor dem Drehen, um eine einwandfreie Uberlagerung der Ansätze zu garantieren. Nach der Rotation reichte die Schwerkraft zur Ausrichtung der Gelschichten quer zur senkrechten Röhrchenachse aus, da die Polymerisation erst 24-30 min nach der Gelbereitung einsetzte. Sie begann wegen der nach oben zunéhmenden Cyanoferratkonzentration im dichten Gel und schloß nach 30-40 min makroskopisch ab, so đaß 12 Röhrchen ohne nachteilige Polymerisationsverzögerung gleichzeitig präpariert werden konnten. Da sịch die analytischen Eigenschaften (Auflösung, Untergrundentfärbung, unterdrückte Bandenkrümmung) im weitporigen Gel durch Lagerung besserten, fand offenbar eine Nachpolymerisation statt. $\mathrm{Da}$ das Gemisch $\mathrm{A}$ vor $B$ bereitet wurde, trat die Reaktionswärme nicht gleichzeitig im gesamtẹn Gel auf, so daß Konvektionsströmungen gering blieben. Die resultierende geringe Schlierenbildung, bei Lufttkühlung gelegentlich zu beobachten, ließ sich durch Eintauchen der Röhrchen in etwa $20^{\circ} \mathrm{C}$ warmes Wasser unterdrücken, während Eiswassser ein 
partielles Ablösen der Gelzylinder vom Röhrchen verursachte und damit zu Störungen bei der Densitometrie führte oder bei zu frühem Eintauchen die Polymerisation verzögerte.

\section{Analy tische Variable}

Für die Auftrennung von Serumproteinen mußte die minimale Polyacrylamid-Konzentration unter $40 \mathrm{~g} / 1$ liegen, um Makroglobuline in das Gel eintreten zu lassen. Dabei erwiesen sich, beurteilt nach Anzahl und Schärfe der Banden, ein Rotationswinkel von $5^{\circ}$ und der benutzte Konzentrationsgradient von 1:6,5 als besonders geeignet. Eine Erhöhung der Endkonzentration auf 263,6 g/l (Gradient 1:7) schärfte die Fraktionen vor dem Albumin, verminderte aber die Auflösung im weitporigen Anteil. Denselben Effekt führte beim beschriebenen System die Verlängerung des Ansatzes B, z. B. auf $42 \mathrm{~mm}$, herbei. Während eine Erhöhung der Polyacrylamidkonzentration über $250 \mathrm{~g} / \mathrm{l}$ für Seren unnötig und mit einer verlängerten Laufzeit verbunden war, ließ sich die Trennschärfe im kathodischen Bereich durch einen pH-Gradienten entscheidend verbessern (Abb. 5). Dazu stellten wir bei sonst gleicher Zusammensetzung den Gelpuffer für Ansatz B mit Schwefelsäure auf pH 7,4
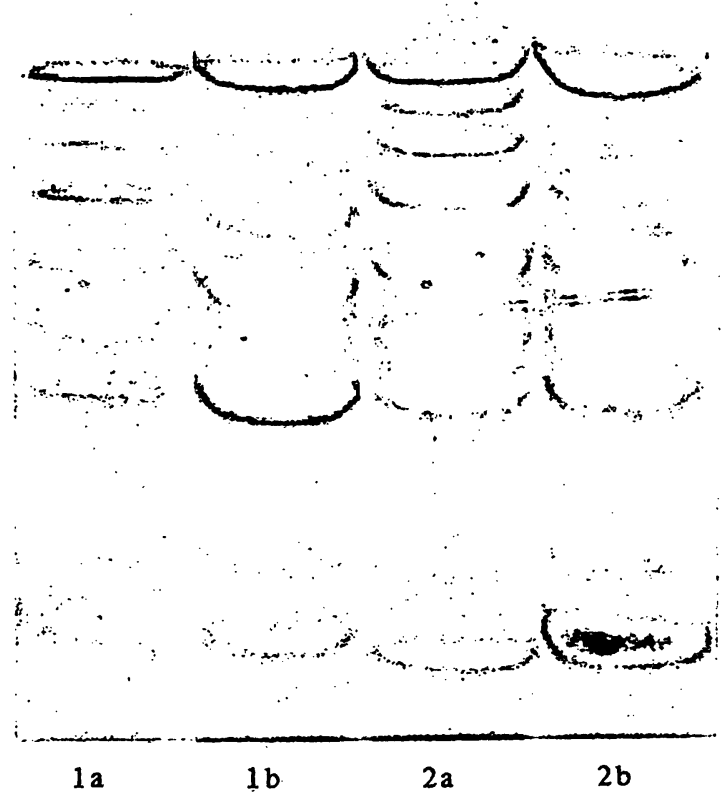

Abb. 5. Proteinpherogramme von 2 Seren in Gradientengelen mit Ornstein-Davis- (Pos. a) und Allen-System (b) bei vierfacher Vergrößerung des weitporigen Anteils $(3 \mu 1$ Serum, Amidoschwarz, Rotationswinkel $5^{\circ}$, oben Kathode).
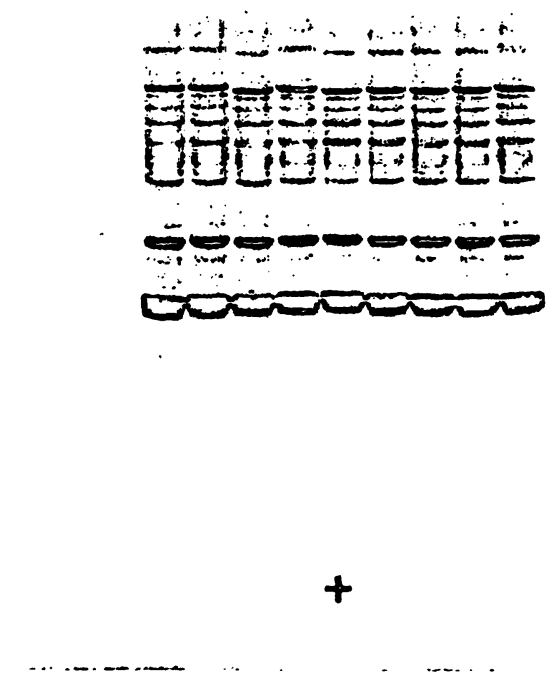

Abb. 6. Proteinpherogramme in Gradientengelen derselben Präparation ( $3 \mu \mathrm{l}$ Serum, Amidoschwarz, OrnsteinDavis-System, Rotationswinkel $5^{\circ}$ ).

ein. Bei einem Rotationswinkel von $5^{\circ}$ ließen sich mit diesem System (Polyacrylamid-Gesamtkonzen tration $37,0-240,7 \mathrm{~g} / \mathrm{l}, \mathrm{pH} 7,4-8,6)$ in Seren 27-30 Proteinbanden trennen (Abb. 6). Für die Darstellung von Lipoproteinen und Enzymen genügten Gradientengele mit einem konstanten $\mathrm{pH}$-Wert (Abb. 7).

Eine Verminderung der Tris-Konzentration im Gelpuffer von 1,5 auf $0,5 \mathrm{~mol} / 1$ verkürzte $z$ war die Trenndauer und beschleunigte die Fixierung der Gele, doch nahm die Trennschärfe durch Verbreiterung der Banden ab. Auch die empfohlene Zugabe von Hydantoin $50 \mathrm{mmol} / \mathrm{l}$ (15) oder Harnstoff $170 \mathrm{mmol} / \mathrm{l} \mathrm{Ansatz}$ beschleunigte in allen Systemen die kathodischen Fraktionen und verschlechterte damit die Auflösung im Bereich der Haptoglobine. Ähnliche Nachteile resultierten aus Vorläufen mit $50 \mathrm{~V}$ über $2 \mathrm{~h}$ zur Entfernung von Persulfat. Dagegen beeinträchtigte Triton X-100 bis zu $10 \mathrm{~g} / 1$ weder die Polymerisation noch die Trennleistung, doch ließ es schon ab $100 \mathrm{mg} / \mathrm{l}$ die Gele während der Elektrophorese aus den Röhrchen treten. Daher wurde es nur mit $10 \mathrm{mg} / 1$ den Ansätzen zugefügt, wenn hochvernetzte Gele (über $250 \mathrm{~g} / \mathrm{l}$ ) aus den Röhrchen gepreßt werden mußten.

Auch die Natur des Gegenions beeinflußte bei gleichem pH-Wert von 8,6 und identischer Tris-Konzentration (165 mmol/l) des Elektrodenpuffers die Trennung im Gradientengel: EDTA (verminderte Trennschärfe und verzögerte Wanderung aller Fraktionen), Borat 


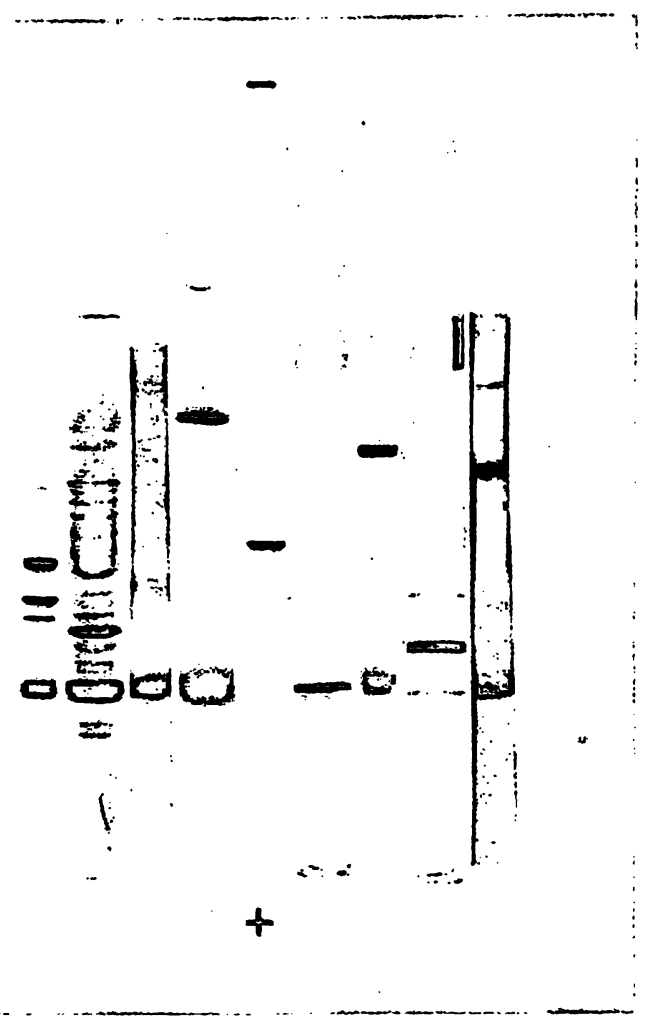

Abb. 7. Pherogramme in Gradientengelen. Die Positionen 2, 4, 6 und 8 enthalten die Gele in Röhrchen. Von links nach rechts: Lactatdehydrogenase-Zymogramm (mit Albuminbande), Proteinpherogramm, Arylamidase (m. A.), Lipopherogramm (m. A.), alkalische Phosphatase, Magensaft, Esterase (Indoxylacetat, m. A.), Liquor, Esterase (Naphthylacetat, m. A.), sämtlich in $5^{\circ}$-Gelen.

(schlechtere Fraktionierung im kathodischen Gelabschnitt) und Glycylglycin (ungenügende anodische Auflösung) waren Glycin eindeutig unterlegen. Dasselbe galt auch für den bei Mikroverfahren empfohlenen Puffer Tris $50 \mathrm{mmol} / \mathrm{l}$ - Glycin $384 \mathrm{mmol} / \mathrm{l} \mathrm{pH} \mathrm{8,4} \mathrm{(4).}$ Andere Puffer wurden nicht getestet.

Während die Konzentration des Elektrodenpuffers und sein $\mathrm{pH}$-Wert dem erreichbaren Optimum en tsprachen, konnten wir die Trennbedingungen mangels Gegenkühlung nicht variieren. Dagegen bedeutete das Serum- volumen von $3 \mu$ den bestmöglichen Kompromiß zwischen Nachweisgrenze und Bandenkrümmung, wobei eine Verdünnung der Probe mit Laufpuffer oder Saccharose $1 \mathrm{~mol} / \mathrm{l}$ nicht erforderlich war. Schließlich wählten wir nach einem Vergleich verschiedener Färbungen das beschriebene Verfahren aus folgenden Gründen: Die Verwendung von Coomassie Brillantblau R250 nach Chrambach et al. (13) oder Weber \& Osborn (16) erforderte eine vorangehende lange Fixierung und beeinträchtigte die Entfärbung des Hintergrundes. Beim Vorgehen nạch Dunker \& Rueckert (17) resultierte bei extrem langer Färbedauer eine gute Entfärbung, aber alle mit Coomassie behandelten Zylinder durften nicht in Entfärbelösung, sondern nur - unter Quellung in Essigsäure aufbewahrt werden. Dagegen konnten wir mit Amidoschwarz, Ponceau und Säureviolett gleichzeitig färben und fixieren, die proteinfreie Gelmatrix farblos erhalten, und die Gele mindestens 6 Monate ohne Absorptionsverlust aufbewahren. Verkürzung der Färbezeit (auch bei Erhöhung der Reaktionstemperatur auf $60^{\circ} \mathrm{C}$ ), Fortlassen vön Sulfosalicylsäure oder Ersetzen durch Trichloressigsäure $1 \mathrm{~mol} / \mathrm{l}$ verminderte die Schärfe der Banden. Uniblue, ein Vinylsulfonderivat des Remazolbrillantblau $R$, konnte mit denselben günstigen Eigenschaften wie Amidoschwarz verwendet und außerdem zur Nachfärbung bei anderen Nachweisen benutzt werden. Bei vergleichender Densitometrie (in den zugehörigen Absorptionsmaxima) der Anfärbung von 1-10 $\mathrm{g}$ Humanalbumin in $2 \mathrm{~mm}$ breiten Gelzonen lieferte Coomassie nur unterhalb von $2 \mu \mathrm{g}$ die höchsten Werte. Über $5 \mu \mathrm{g}$ lagen die Extinktionen mit den anderen Farbstoffen hingegen 2 bis 5 mal höher.

\section{Pherogramme}

Bei der gleichzeitigen Elektrophorese eines Normalserums in 12 Gelen derselben Herstellung. $\left(5^{\circ}\right.$ Neigungswinkel) variierte die Distanz der Albuminbande vom Start maximal zwischen 47 und $49 \mathrm{~mm}$ (Abb. 5), bei 10 Läufen mit 120 Gelen zwischen 47 und $51 \mathrm{~mm}$, während die Länge der Gelzylinder nach der Entfärbung zwischen 74 und $76 \mathrm{~mm}$ betrug. Eine ähnlich gute Reproduzierbarkeit ergab sich auch für den Abstand Präalbumin $-\alpha_{2}$ -

Tab. 2. Einfluß des Rotationswinkels auf die Eigenschaften von Gradientengelen mit 37,0-240,7 g/l Gesamtkonżentration an Polyacrylamid, pH 8,6 (Allen-System). Densitometrie, s. Methoden; Proteinpherogramme aus einem Poolserum ( $3 \mu \mathrm{l}$, Amidoschwarz, Essigsäure-Methanol). Angabe von $\bar{x} \pm 2 \mathrm{~s}$ bei 12 (gemeinsame Präparation) und 120 Gelen (10 Präparationen).

\begin{tabular}{|c|c|c|c|c|c|c|}
\hline \multirow[b]{3}{*}{ Untersuchte Parameter } & \multicolumn{6}{|c|}{ Rotationswinkel der Röhrchen } \\
\hline & $3^{\circ}$ & $3^{\circ}$ & $5^{\circ}$ & $5^{\circ}$ & $10^{\circ}$ & $10^{\circ}$ \\
\hline & 12 & 120 & 12 & 120 & 12 & 120 \\
\hline $\begin{array}{l}\text { Steigung der densito- } \\
\text { metrischen Absorptions- } \\
\text { geraden in }\end{array}$ & $46 \pm 2$ & $46 \pm 5$ & $61 \pm 1$ & $61 \pm 3$ & $76 \pm 1$ & $76 \pm 2$ \\
\hline $\begin{array}{l}\text { Länge des linearen } \\
\text { Gradienten in mm }\end{array}$ & $62 \pm 5$ & $62 \pm 8$ & $43 \pm 3$ & $43 \pm 7$ & $22 \pm 2$ & $22 \pm 3$ \\
\hline $\begin{array}{l}\text { Abstand Präalbumin - } \\
\alpha_{2} \text {-Makroglobulin in mm }\end{array}$ & $59 \pm 4$ & $59 \pm 7$ & $33 \pm 2$ & $33 \pm 4$ & $21 \pm 2$ & $21 \pm 2$ \\
\hline Anzahl der Fraktionen & & end & & & & \\
\hline
\end{tabular}




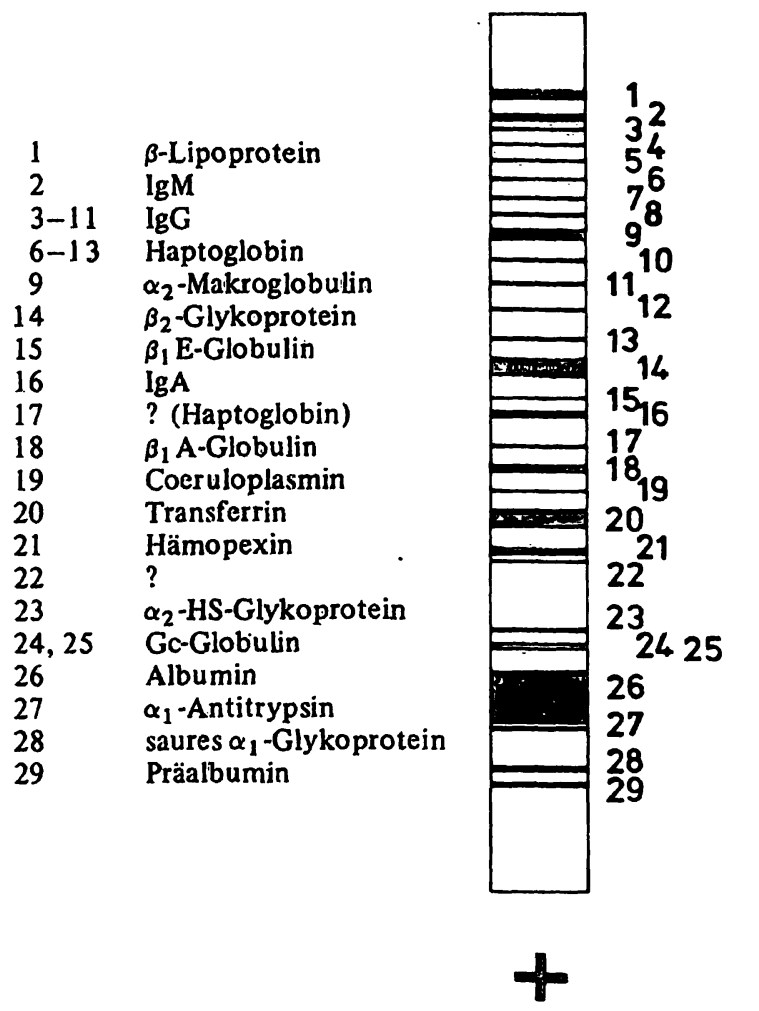

Abb. 8. Lage der wichtigsten Serumproteine im Gradientengel bei immunologischer Zuordnung. Polyacrylamid-Gesamtkonzentration $37-240,7 \mathrm{~g} / 1$, Rotationswinkel $5^{\circ}, \mathrm{pH} 7,4-8,6$.

Makroglobulin (Tab. 2) und damit für die Lokalisation aller Fraktionen. Trotz Einsatz von $20 \mu \mathrm{l}$ Serum gelang uns keine vollständige Identifizierung, da nicht sämtliche Antiseren zur Verfügung standen. Die Reihenfolge der Banden war nach $16 \mathrm{~h}$ Trenndauer immer gut erkennbar und entsprach (Abb. 8) nahezu den Angaben von Hoffmeister (18).

Mit der gleichen Konstanz ließen sich Zymogramme erhalten. Bei störender Albuminmarkierung durch Bromphenolblau, z. B. beim Nachweis von Lactatdehydrogenase (Abb. 7) konnte es durch Fluorescein ersetzt werden (15). Zwei kathodisch gelegene Banden mit $\alpha$-Amylaseaktivität stellten wir nach Davies (19) durch $12-16 \mathrm{~h}$ Inkubation in einer Suspension von Cibachronblau F3AStärke (Phadebas Amylase Test) dar. Dagegen mißlang der Nachweis von $\gamma$-Glutamyltransferase nach modifizierten (Glycylglycinpuffer, $\mathrm{pH}$, Diazoniumverbindungen) und Originalmethoden für Folien (20) oder Agar (21). Verglichen mit normalen Verfahren waren die enzymaktiven Banden von Esterase, Arylamidase, alkalischer Phosphatase und Lactatdehydrogenase im Gradientengel deutlich schmaler (Abb. 7).

\section{Diskussion}

Gegenüber den üblichen Methoden zur Gradien tengelbereitung mit Apparaturen zur Füllung einzelner (22) oder mehrerer Rund- (23), Platten- $(1,2,3,24,25)$ oder Mikrogele (26) verzichtete das angegebene Verfahren auf eine Polymerisationsverzögerung. Auch der Zusatz von Saccharose $(2,3)$, der über einen Viskositätsgradienten die Ferguson-Beziehung zwischen Molekulargewicht und Laufstrecke verändert (27), war entbehrlich. Damit entfielen auch technische Probleme, die nach Kapadia et al. (27) sonst bei der Herstellung reproduzierbarer Gelgradienten auftreten: Konvektionsströme und wechselnde Polymerisationsgeschwindigkeit als Folge der mit steigender Konzentration zunehmenden Reaktionswärme, eine geringe und im Verlauf dès Gradienten unterschiedlich wirksame Polymerisation als Ergebnis verzögernder Bedingungen, verlängerter Zutritt von Licht und Sauerstoff zum Gelgemisch und Schwierigkeiten bei der Temperaturkontrolle. Andererseits erlaubte die Benutzung der Drehvorrichtung nur die Herstellung linearer Gradienten, während Mischapparate auch andere Gradienten erzeugen und in besonderen Fällen (25) die BIS-Konzentration zusätzlich zum variablen Acrylamidverhältnis verändern können.

Dagegen ermöglichte das Rotationsprinzip die Anwendung vieler Techniken. Vor allem ließ die optische Kontrolle des Gradienten durch einen stabilen anodisch wandernden Farbstoff die Polyacrylamidkonzentration in jedem Gelsegment bestimmen und nicht allein die Form des Gradienten, die auch mit ungeladenen Farbstoffen darzustellen ist (26), beurteilen. Dies gestattete, die spätere Position eines Proteins von bekanntem Molekulargewicht vorherzusagen und die erforderlichen Polyacrylamidkonzentrationen für alle Trennprobleme zu berechnen. Im Gegensatz zur Molekulargewichtsbestimmung, die man besser mit einem pH-kontinuierlichen System nach Allen durchführt (28), trennt man die Serumproteine mit einem zusätzlichen pH-Gradienten (analog Ornstein \& Davis, 29) durch den Konzentrationseffekt im weitporigen Gel optimal. Obwohl bei der benutzten Laufzeit kein „dead stop" der Fraktionen eintrat und ihre Reihenfolge nicht dem Verhältnis der Molekulargewichte entsprach, beeinflußte eine Veränderung der Trenndauer um $\pm 2 \mathrm{~h}$ die gute Reproduzierbarkeit der Ergebnisse nicht. Wir verzichteten daher auch auf eine systematische Untersuchung verschiedener $\mathrm{pH}$-Gradienten, die sich mit Indikatorfarbstoffen darstellen und möglicherweise eine beschleunigte Fraktionierung zulassen. 


\section{Literatur}

1. Slater, G. G. (1968), Anal. Biochem. 24, 215-217.

2. Margolis, J. \& Kenrick, K. G. (1968), Anal. Biochem. 25, $347-362$.

3. Margolis, J. \& Kenrick, K. G. (1967), Nature (London) 214, 1334-1336.

4. Rüchel, R., Mesecke, S., Wolfrum. D. I. \& Neuhoff, V. (1973), Hoppe-Seyler's Z. Physiol. Chem. 354, 1351-1368.

5. Polyacrylamide gradient gels PAA 4/30 (1974), Pharmacia Fine Chemicals AB, Uppsala, Sweden.

6. Gradipore Gels (1972), Gradients Pty. Ltd., Lane Cove, Australia.

7. Schwartz, A. N. \& Zabin, B. A. (1966), Anal. Biochem. 14 $321-327$.

8. Allen, R. C. (1974), in Electrophoresis and Isoelectric Focusing in Polyacrylamide Gel (Allen, R. C. \& Maurer, H. R., eds.), p. 289, Verlag Walter de Gruyter \& Co., Berlin-New York.

9. Dietz, A. A. \& Lubrano, T. (1967), Anal. Biochem. 20, 246-257.

10. Lorentz, K., Flatter, B. \& Heydrich, D. (1974), diese Z. 12 , $81-86$.

11. Lorentz, K., Marunowski, A. \& Ritter, U. (1974), diese Z. 12, 468-473.

12. Datyner, A. \& Finnimore, E. (1973), Anal. Biochem. 52, 45-55.

13. Chrambach, A., Reisfeld, R. A., Wyckoff, M. \& Zaccari, J. (1967), Anal. Biochem. 20, 150-154.

14. Abraham, K., Schütt, K., Müller, l. \& Hoffmeister, H. (1970), diese Z. 8, 92-98.

15. Neuhoff, V. (1973), Micromethods in Molecular Biology, p. 14, Springer-Verlag, Berlin-Heidelberg-New York.

16. Weber, K. \& Osbom, M. (1969), J. Biol. Chem. 244, 4406-4412.
17. Dunker, A. K. \& Rueckert, R. R. (1969), J. Biol. Chem. 244. 5074-5080.

18. Hoffmeister, H. (1974), in Electrophoresis and Isoelectric Focusing in Polyacrylamide Gel (Allen, R. C. \& Maurer, H. R., eds.), p. 266-278, Verlag Walter de Gruyter \& Co., Berlin-New York.

19. Davies, T. J. (1972), J. Clin. Pathol. 25, 266-267.

20. Patel, S. \& O'Gorman, P. (1973), Clin. Chim. Acta 49, $11-17$.

21. Miyazaki, S. \& Okumura, M. (1972), Clin. Chim. Acta 40,193-197.

22. Kidby, D. K. (1970), Anal. Biochem. 34, 478-484.

23. Caton, J. E. \& Goldstein, G. (1971), Anal. Biochem. 42, $14-20$.

24. Margolis, J. (1969), Anal. Biochem. 27, 319-322.

25. Margolis, J. \& Wrigley, C. W. (1975), J. Chromatogr. 106, 204-209.

26. Dames, E. \& Maurer, H. R. (1974), in Electrophoresis and Isoelectric. Focusing in Polyacrylamide Gel (Allen, R. C. \& Maurer, H. R., eds.), p. $221-.231$, Verlag Waltet de Gruyter \& Co., Berlin-New York.

27. Kapadia, G., Chrambach, A. \& Rodbard, D. (1974), in Electrophoresis and Isoelectric Focusing in Polyacrylamide Gel (Allen, R. C. \& Maurer, H. R., eds.), p. 127-128, Verlag Walter de Gruyter \& Co., Berlin-New York.

28. Lorentz, K. (1976), Anal. Biochem. 76, 214-220.

29. Maurer, H. R. (1971), Disc Electrophoresis and Related Techniques of Polyacrylamide Gel Electrophoresis, p. 22-32, Verlag Walter de Gruyter \& Co., Berlin-New York.

Prof. Dr. K. Lorentz Kronsforder Allee 71/72 D-2400 Lübeck 